\title{
Effects of Reinforcement Rate on the Aversive Function of Timeout from Positive Reinforcement
}

Cory Whirtley

cwhirtle@mix.wvu.edu

Follow this and additional works at: https://researchrepository.wvu.edu/etd

Part of the Experimental Analysis of Behavior Commons

\section{Recommended Citation}

Whirtley, Cory, "Effects of Reinforcement Rate on the Aversive Function of Timeout from Positive Reinforcement" (2018). Graduate Theses, Dissertations, and Problem Reports. 3740.

https://researchrepository.wvu.edu/etd/3740

This Thesis is protected by copyright and/or related rights. It has been brought to you by the The Research Repository @ WVU with permission from the rights-holder(s). You are free to use this Thesis in any way that is permitted by the copyright and related rights legislation that applies to your use. For other uses you must obtain permission from the rights-holder(s) directly, unless additional rights are indicated by a Creative Commons license in the record and/ or on the work itself. This Thesis has been accepted for inclusion in WVU Graduate Theses, Dissertations, and Problem Reports collection by an authorized administrator of The Research Repository @ WVU. For more information, please contact researchrepository@mail.wvu.edu. 


\title{
Effects of Reinforcement Rate on the Aversive Function of Timeout
} from Positive Reinforcement

\author{
Cory Whirtley \\ Thesis Submitted to the \\ Eberly College of Arts and Sciences \\ at West Virginia University
}

in partial fulfillment of the requirements for the degree of

\author{
Master of Science \\ in \\ Psychology
}

\author{
Michael Perone, Ph.D., Chair \\ Claire C. St. Peter, Ph.D., BCBA-D \\ Cole Vonder Haar, Ph.D. \\ Department of Psychology
}

Morgantown, West Virginia

2018

Keywords: timeout; timeout from positive reinforcement; reinforcement rate; punishment; rats Copyright 2018 Cory Whirtley 


\section{ABSTRACT \\ Effects of Reinforcement Rate on the Aversive Function of Timeout from Positive Reinforcement}

\section{Cory Whirtley}

Although the use of timeout from positive reinforcement is widespread and has been shown to be an effective punisher in clinical settings, the factors responsible for its effectiveness are not fully understood. The present experiment was designed to evaluate one of these factors, the reinforcement rate underway during periods of time-in. Rats' lever pressing was maintained on variable-interval schedules of food reinforcement in a multiple schedule with two components. In one component, no timeouts were delivered. In the other component, when a variable-ratio schedule was met, lever presses were followed by a 30-s timeout during which a tone sounded, the component light was extinguished, and the food schedule was suspended. Across conditions, the reinforcement rates in both components ranged from 0.5 to 6 pellets per min and the degree of response suppression was evaluated. In the leanest condition, 0.5 pellets per min, the timeouts failed to suppress responding. Generally, as the reinforcement rate was increased, the degree of suppression was enhanced systematically, showing that the punitive function of timeout is directly related to time-in reinforcement rate. The results are discussed in relation to laboratory research on timeout avoidance and shock punishment. 


\section{Acknowledgements}

I would like to thank Mike Perone for his mentorship and guidance during all of our explorations of timeout. I am grateful that Mike saw my potential as an undergraduate and offered me the opportunity to work on our first timeout study. I would also like to thank my committee members - Mike Perone, Claire St. Peter, and Cole Vonder Haar - for their helpful comments and suggestions on my document.

Many thanks go to the members of the Perone lab for helping me conduct the sessions some of which seemed like they would never end.

I thank all of my friends and family for their continued support. Special thanks go to my fiancé and laboratory copilot, Forrest Toegel, for his continuous support and ability to endure longwinded rants about timeout. Without his love and friendship, this project would have been a lot less fun. 


\section{Table of Contents}

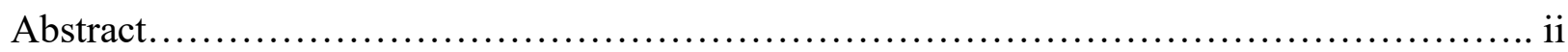

Acknowledgements................................................................

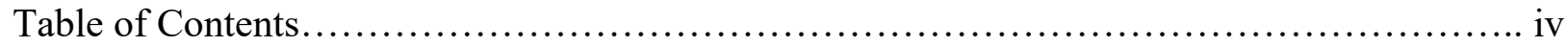

List of Tables......................................................................

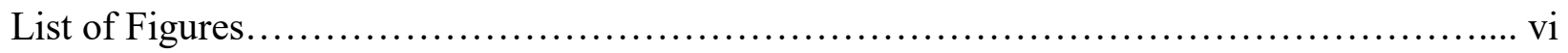

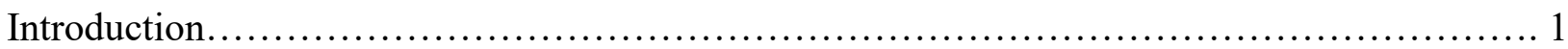

Method.......................................................................... 8

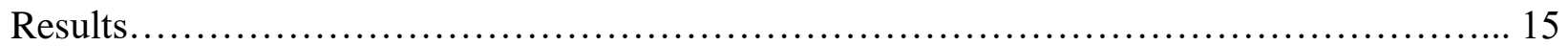

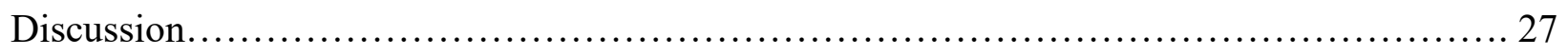

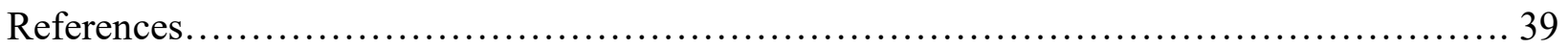




\section{List of Tables}

Table 1. The experimental ( 0.5 to 6 pellet deliveries per min) conditions (in order, left to right) and the number of sessions in each.

Table 2. Obtained pellet delivery rates in relation to the programmed rates across the baseline (BL) and punishment (PUN) components for each experimental condition. The conditions are shown in order of occurrence (left to right). The rates were aggregated (total number of pellets/total time spent in time-in) across the six stable sessions for each condition............ 16

Table 3. The duration of time-in for the baseline and punishment components for each condition. All values are means (standard deviations in parentheses) over the last six sessions of each condition. The rightmost column shows the minimum required duration of time-in (Minimum Duration) that had to occur in each component per session. The minimum was determined by the

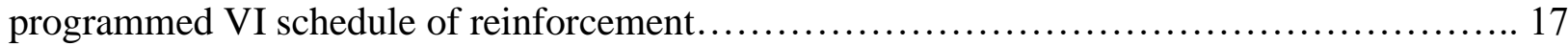

Table 4. Timeout rate (timeouts per min), total timeouts, and timeout duration (min). All values are means (standard deviations in parentheses) over the last six sessions of each condition...... 21 


\section{List of Figures}

Figure 1. Mean response rates during the baseline (BL) and punishment (PUN) components (left column), mean suppression ratios (middle column), and mean discrimination ratios (right column) as a function of the pellet delivery rate. All error bars extend 1 SD above and below the mean; in some cases, the error bars are not visible because the data point overlaps them entirely. In some cases, the data points from the replications overlap the initial condition entirely (grey squares). Results are from the last six sessions of each condition............................ 19

Figure 2. Median post-timeout latencies (s) as a function of the pellet delivery rate (left column) and median post-reinforcer latencies (s) during the baseline (BL) and punishment (PUN) components as a function of the pellet delivery rate (middle column). All error bars extend from the $25^{\text {th }}$ to $75^{\text {th }}$ percentiles. In some cases, the data points from the replications overlap the initial condition entirely.

Figure 3. Median post-timeout latencies (s) as a function of consecutive timeouts. Timeouts are considered consecutive when no reinforcer occurs between the offset of one timeout and the onset of the next timeout. The panel in the lower right-hand corner shows the goodness of fit for each of the lines fitted to the results from each condition.

Figure 4. Response strength as a function of reinforcement rate. The dotted line represents the contributions of reinforcement to response strength. The dashed line represents the contributions of timeouts to response strength. The solid line represents the net effect of both reinforcement

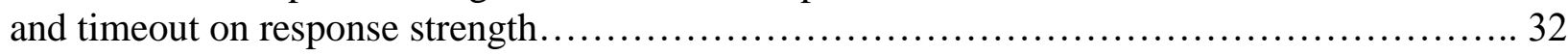




\section{Introduction}

Over the last four decades, the procedure known as timeout from positive reinforcement (hereafter, "timeout") has been the most widely used punishment technique to reduce problem behavior for persons with developmental disabilities (Lydon, Healy, Moran, \& Foody, 2015). Timeout has been demonstrated to work with other populations, including normally developing young children (Larzelere, Schneider, Larson, \& Pike, 1996; Mathews, Friman, Barone, Ross, \& Christopherson, 1987), older children with attentiondeficit/hyperactivity disorder (ADHD; Fabiano et al., 2004), and adolescent psychiatric patients (Crespi, 1988). Timeout has been shown to reduce a wide range of problem behavior, such as tantrums (Porterfield, Herbert-Jackson, \& Risley, 1976), classroom disruptions (Donaldson \& Vollmer, 2012), and aggression (Benjamin, Mazzarins, \& Hupfersmid, 1983). In a recent survey of educators associated with the Council for Children with Behavioral Disorders, 17 percent reported using a timeout procedure at least once per day and 29 percent reported using the procedure one to four times per week (Jones, 2015).

In a typical timeout procedure, a target response is followed by a signaled period without positive reinforcement (Azrin \& Holz, 1966). For example, Donaldson (2011) implemented a timeout procedure as a clinical intervention to decrease children's problem behavior during free play on the playground or in the home. Problem behavior was defined as any instance of rule-breaking in the current setting (e.g., pushing peers, throwing toys). A timeout followed each instance of problem behavior: The child was removed from the freeplay situation, confined to an isolated area for the duration of the timeout, and then released back into free play. The timeouts reduced problem behavior below baseline levels, an effect commonly reported in clinical settings (Brantner \& Doherty, 1983; Everett, Hupp, \& Olmi, 
2010).

Although timeout procedures are widely used in clinical interventions, the conditions under which timeout will reduce problem behavior are not fully understood. It is difficult to uncover these conditions within the clinical setting. This may not be surprising, as timeout (and other punishment) procedures are often chosen as a clinical intervention when the reinforcer maintaining the problem behavior cannot be withheld or controlled (Lerman \& Vorndran, 2002). For example, consider cases in which the delivery of social positive reinforcers (e.g., attention) are found to maintain problem behavior, a common assessment result in clinical settings (Beavers, Iwata, \& Lerman, 2013). If the frequency or severity of the problem behavior is high, it may be difficult or impossible to remove social reinforcers completely or consistently in an uncontrolled environment such as a classroom. In these cases, a timeout procedure is chosen because the reductive effects of the timeouts are expected to compete with the strengthening effects of the social reinforcement (Lerman \& Vorndran, 2002). With this intervention, the clinician makes a series of choices that permit tight control over the parameters of timeout delivery and the timeout environment (e.g., schedule, location, and duration of timeout; see Everett et al., 2010 for a review). The rest of the environment which includes the reinforcing consequences of the problem behavior - is typically left uncontrolled.

From an ethical standpoint, clinicians should aim to use the most effective and least intrusive timeout procedure (Van Houten et al., 1988). The conditions under which clinicians can expect the procedure to be most effective has not been the object of much controlled research. In the absence of research on timeout, several rules of thumb (e.g., timeouts should last 1 min per year of child's developmental age) have guided the clinical application of 
timeout procedures (Hackenberg \& Defulio, 2007). One common guideline suggests that clinicians can enhance the effects of timeout by enriching the "time-in" environment - that is, the environment in which reinforcement for the problem behavior is available (Shriver \& Allen, 1996). Unfortunately, research providing direct support for Shriver and Allen's guideline has been limited to only one empirical study, discussed below.

Solnick, Rincover, and Peterson (1977) studied the effects of timeout on the problem behavior of a boy with Down's syndrome when the time-in environment was either impoverished or enriched. During time-in, reinforcers such as toys were available following the completion of simple tasks. During impoverished time-in periods, the reinforcers were simple toys (i.e., a rubber ball, a small block). During enriched time-in periods, more complex reinforcers were available, such as social attention and a variety of novel toys (e.g., musical instruments, jack-in-the-box). In the experimental conditions, any instance of self-injury or spitting produced a 90-s timeout, during which any ongoing music, toys, and attention were removed. The impoverished and enriched conditions alternated multiple times. In baseline, spitting was maintained at approximately 60 instances per session and self-injury was maintained at approximately 120 instances per session. Timeouts from the enriched environment immediately reduced problem behavior to near-zero levels, whereas timeouts from the impoverished environments were less successful: Self-injury was maintained at approximately 80 instances per session in the first exposure, 8 instances per session in the second exposure, and near-zero levels in the third exposure. Solnick et al.'s findings are consistent with Shriver and Allen's (1996) recommendation that timeouts should be more effective when the rate of reinforcement during the time-in environment is raised. More broadly, Solnick et al.'s study suggests that the characteristics of the time-in environment may 
determine in part the behavioral function of timeout.

Although direct evidence for the enrichment guideline in clinical settings is limited to one study (Solnick et al., 1977), theoretical support comes from Fantino's (1977) delayreduction hypothesis. Fantino proposed that stimuli correlated with reductions in the reinforcement rate should acquire aversive properties, and that stimuli correlated with greater reductions in reinforcement rate should be more aversive than stimuli correlated with smaller reductions. Because timeouts from an enriched environment would produce greater reductions from the prevailing reinforcement rate, timeouts from an enriched environment should be more aversive than timeouts from an impoverished environment. To assess the contributions of reinforcement rate without the variability found in clinical settings, it may be productive to study timeout in a controlled, experimental environment. I reviewed the literature on timeout punishment in search of basic experiments that directly studied the effects of reinforcement rate during time-in on the punishing efficacy of timeout. Behavioral journals, such as the Journal of the Experimental Analysis of Behavior, Journal of Applied Behavior Analysis, The Psychological Record, and Behavior Analysis in Practice were searched. In addition, search engines, such as EBSCOhost, PubMed, and Google Scholar were used. The reference sections of all relevant papers were reviewed. For all searches, the following key words were used alone or in combination: timeout, time-out, time out, punishment, negative punishment, aversion conditioning, isolation, and conditioned suppression. To date, no basic experiments that directly studied the effects of reinforcement rate on timeout punishment could be found.

One study by Kaufman and Baron (1968), however, manipulated the reinforcement rate indirectly. Instead of altering the programmed reinforcement rate, they manipulated a 
variable related to the value of reinforcement. They restricted rats' access to food to establish body weights at specific percentages of the free- feeding weight. As body weights are lowered, the value of food reinforcers should increase, thus enriching the time-in environment (Michael, 1982). Kaufman and Baron trained their rats to press a lever in a three-response sequence. Response 1 and Response 2 both were reinforced with a 0.1-ml dipper of evaporated milk; Response 3 produced a 2-min timeout signaled by a tone. To measure the effect of the timeouts, Kaufman and Baron compared the time between Responses 2 and 3 with the time from the start of the sequence to Response 1 and the time between Responses 1 and 2 . The third time was elevated relative to the first two, indicating the punitive effect of the timeout that followed every third response.

After a baseline condition in which body weights were 80 percent of each rat's freefeeding weight, the weights were raised or lowered. Surprisingly, lowering body weights from 80 to 70 percent reduced the punitive effect of timeout, as shown by reductions in the third interresponse times. Raising weights from 80 to 90 percent increased the punitive effect, as shown by increased interresponse times. The manipulation that presumably increased the value of the reinforcement reduced the punishing efficacy of timeout from that reinforcement. Kaufman and Baron (1968) manipulated the reinforcement rate indirectly by altering deprivation levels. Basic research on timeout punishment has not yet answered what will happen if the reinforcement rate is altered directly. There are several studies, however, that have examined this relation with timeout avoidance procedures. In these studies, the aversiveness of a timeout is measured in terms of the rate of a response that avoids upcoming timeouts (Leitenberg, 1965).

Two avoidance studies (Thomas, 1964; van Haaren \& Anderson, 1998) obtained 
results that contradict Fantino's (1977) hypothesis and Solnick et al.'s (1977) findings. van Haaren and Anderson (1998) compared the effects of two rates of free food deliveries on the aversiveness of timeout. Rats were trained on a multiple schedule with two components. In the component designated as "rich", food pellets were delivered independently of responding after random periods of time averaging $30 \mathrm{~s}$. This component was signaled by the presence of a tone. In the "lean" component, the average time between pellets was $120 \mathrm{~s}$, and the signal was the absence of the tone. A 50-s timeout, signaled by the offset of the chamber lights, was delivered every $10 \mathrm{~s}$ unless the rat pressed a lever, in which case the timeout was postponed for $20 \mathrm{~s}$. If all lever presses were spaced less than $20 \mathrm{~s}$ apart, all timeouts could be avoided. Four of the five rats avoided the timeous at higher rates during the lean component than during the rich component $(34 \%, 50 \%, 18 \%$, and $36 \%$ higher in the lean). When the rate of reinforcement was raised, the rate of avoidance responding decreased - an inverse relation between the density of the food deliveries and the aversiveness of timeout.

This inverse relation was also reported by Thomas (1964). Pigeon's key pecks were maintained on a variable-interval (VI) schedule in which a food reinforcer was delivered following the first response after a variable amount of time elapsed. Across conditions, the rate of reinforcement was either relatively lean (VI 9-min) or relatively rich (VI 1-min). A 5-min timeout was delivered every $30 \mathrm{~s}$ unless the pigeon pecked a separate avoidance key, in which case the timeout was postponed for $60 \mathrm{~s}$. If all pecks were spaced less than $60 \mathrm{~s}$ apart, all timeouts could be avoided. Responding on the avoidance key was maintained at about 2.5 responses per min during the VI 9-min conditions and fell to near zero during the VI 1-min conditions.

The findings from Thomas (1964) and van Haaren and Anderson (1998) contradict the 
hypothesis that timeouts should be more aversive when the prevailing environment is enriched (Fantino, 1977; Solnick et al., 1972). Instead, timeouts from lean schedules of reinforcement appear be more aversive than timeouts from rich schedules. A major limitation of these studies is that comparisons were made between only two schedule values. Two later studies were designed to evaluate the function across a wider range of reinforcement rates, and they found that avoidance responding increased as the rate of reinforcement was raised, that is, they reported a direct relation between the rate of reinforcement and the aversiveness of timeout (Holtyn, 2012; Richardson \& Baron, 2008).

Richardson and Baron (2008, Experiment 1) delivered food pellets to rats independently of responding at variable times (VT schedule). The rate of pellet deliveries was manipulated across conditions: $0.125,0.25,0.5$, 1 , and 2 pellets per min. A 30 -s timeout, signaled by the retraction of the response lever and offset of the chamber lights, was delivered every $30 \mathrm{~s}$ unless the rat pressed the avoidance lever, in which case the timeout was postponed for $30 \mathrm{~s}$. If all lever presses were spaced less than $30 \mathrm{~s}$ apart, all timeouts could be avoided. In general, responding increased as the rate of pellet delivery was raised, indicating that the aversiveness of timeout was directly related to the rate of food delivery.

Holtyn (2012, Experiment 1) systematically replicated Richardson and Baron's (2008) procedure with deliveries of food pellets and sucrose water as the response-independent events during time-in. The rate of pellet deliveries $(0.125,0.25,0.5,1$, and 2 pellets per min) was the same as in Richardson and Baron's study. A 30-s timeout, signaled by the retraction of the lever and offset of the chamber lights and white noise, was delivered every $30 \mathrm{~s}$ unless the rat responded, in which case the timeout was postponed for 30 s. As in Richardson and Baron's study, avoidance rates were a direct function of the delivery rates. 
Richardson and Baron (2008) and Holtyn (2012) evaluated a wide range of delivery rates and found that the aversiveness of timeout was a direct function of the delivery rates. One may reasonably infer that if timeout from food in an avoidance paradigm functions as an aversive stimulus and the aversiveness of the timeouts is directly related to the rate of food delivery during time-in, then a similar function would be expected to be found in a punishment paradigm. The present research was designed to test this inference.

A two-component multiple schedule was used to evaluate the aversiveness of timeout from reinforcement across a wide range of reinforcement rates. In the baseline component, rats' lever presses were reinforced with food pellets on a VI schedule. In the punishment component, a schedule of timeout punishment was superimposed on the VI schedule. Across conditions, the programmed rates of reinforcement were $0.5,1,2,3$, and 6 pellets per min. If the effects of enriching the time-in environment in a punishment paradigm is parallel to the effects in studies of avoidance (Holtyn, 2012; Richardson \& Baron, 2008), then suppression of responding in the punishment component should be a direct function of the reinforcement rate.

\section{Method}

\section{Subjects}

Six male albino Sprague Dawley rats (Rattus norvegicus) with previous lever-press training were housed in pairs under a 12:12 hr reversed light/dark cycle in a temperaturecontrolled room. Because one rat became ill during the first experimental condition, the veterinary staff removed it from the study and its data are not reported. Experimental sessions were conducted during the dark part of the cycle. The rats were maintained at $80 \%( \pm 2 \%)$ of their free-feeding body weights. Water was freely available in the home cage. Grain-based food 
pellets were delivered as reinforcers during the sessions and grain-blend chow was provided as necessary at least $30 \mathrm{~min}$ after sessions. The treatment of the rats, in and out of the experimental sessions, complied with a protocol approved by the West Virginia University Animal Care and Use Committee.

\section{Apparatus}

Sessions were conducted in four operant-conditioning chambers enclosed in ventilated sound attenuating chests (Med Associates Inc.). General illumination was provided by a 28-V houselight (No. 1820 bulb) located on the back wall. Extraneous sounds were masked by white noise $(80 \mathrm{~dB})$ provided through a 3.5-in speaker located behind the back wall. The interior of each chamber was $29 \mathrm{~cm}$ long, $22 \mathrm{~cm}$ high, and $24 \mathrm{~cm}$ deep. The ceiling and sidewalls were constructed of Plexiglas and the end walls of stainless steel. The floor consisted of 19 stainlesssteel rods $0.5 \mathrm{~cm}$ in diameter spaced approximately $1.3 \mathrm{~cm}$ apart (center to center). Two retractable levers were located on the front wall. Each lever was $4.4 \mathrm{~cm}$ wide, $1.3 \mathrm{~cm}$ thick, and protruded $1.9 \mathrm{~cm}$ into the chamber when inserted. The inside edges of the levers were spaced $11.4 \mathrm{~cm}$ apart $(5.7 \mathrm{~cm}$ from the middle of the wall). The tops of the levers were positioned $8 \mathrm{~cm}$ from the floor. Each lever press produced an audible click as a feedback stimulus from a $28-\mathrm{V}$ relay mounted behind the front wall. Lever presses were reinforced by food pellets (45 mg,

BioServ) delivered into a $5.1 \mathrm{~cm}$ by $5.1 \mathrm{~cm}$ opening centered between the levers on the front wall $5.7 \mathrm{~cm}$ above the grid floor. Each food reinforcer was accompanied by offset of the white noise and onset of a $12-\mathrm{Hz}$ tone for $1 \mathrm{~s}$. Approximately $5 \mathrm{~cm}$ above each lever was a white cue light (No. 1820 bulb). Experimental events were controlled and behavior was recorded with computers running programs written in Visual Basic 2010; the computers were connected to the chambers via digital interfaces (Measurement Computing, model PCI-PDIS08). 


\section{General Procedure}

Sessions were conducted six or seven days per week at approximately the same time each day. After the rat was placed in the chamber, a 5-min delay preceded the start of the session to allow the rat to recover from any effects of handling. During this delay, all chamber lights and sounds were off and both levers were retracted. Sessions began with the illumination of the houselight, onset of the white noise, and the insertion of the right or left lever into the chamber. The houselight remained lit throughout the entire session. Except when noted, each session ended after 60 reinforcers were delivered.

Across all training and experimental conditions, reinforcers were delivered according to VI schedules of reinforcement. In experimental conditions, timeouts were delivered according to a variable-ratio (VR) schedule, which arranged for the timeouts to be delivered following a specified number of responses, on average. The programmed intervals for the VI schedules and the ratio values for the VR schedules were derived from the distribution described by Fleshler and Hoffman (1962).

\section{Preliminary Training}

Lever pressing. All rats had previous experience pressing levers in an undergraduate laboratory course. To facilitate lever pressing on both levers in the present experimental chambers, one lever was inserted into the chamber, the cue light above it was turned on, and every lever press was reinforced. The two levers were alternated after every 10 reinforcers until 100 reinforcers were earned.

Multiple-schedule training. Control of responding was transferred to a multiple VI-VI schedule of food reinforcement. In the first component, one of the levers was inserted into the chamber and the cue light above it was turned on. At the outset of training, presses on this lever 
were reinforced with food according to a VI 5-s schedule. In the second component, the lever used in the first component was retracted and its associated cue light turned off; the other lever was inserted and the cue light above it was turned on. Presses on this lever were reinforced according to a VI schedule with the same mean interval as the first component. Each schedule requirement was in effect for at least one full session. Each component lasted until six pellets were delivered. The two components were alternated until each had been presented five times. Across sessions, the mean interval was raised in steps of $5 \mathrm{~s}$ or $10 \mathrm{~s}$ until it reached $30 \mathrm{~s}$.

When responding in both components met the stability criteria described below (45-59 sessions, depending on the rat), lever bias was assessed by comparing the mean response rate on each lever during the last six sessions. The lever with the higher rate was designated as the "timeout lever" to establish a relatively high baseline rate of responding against which the punitive function of the timeouts could be assessed. The right lever served as the timeout lever for three of the rats (CT29, CT31, CT33) and the left lever served as the timeout lever for the other two rats (CT30, CT32).

Discrimination training. A three-component multiple schedule was used to establish a discrimination between periods of time-in and timeout. The first two components (VI components) were identical to the components in the multiple schedule training just described. The third component consisted of a timeout from the VI schedules. The stimulus conditions and contingencies were the same as those programmed during the response-contingent timeouts in the experiment proper (described below). At the start of the third component, the lever to be used as the timeout lever was inserted into the chamber, the cue light above it was turned off, and the white noise was replaced with a 1000-Hz tone. Pressing the lever, although recorded, was not reinforced. The measurement of these responses was used to assess the discrimination between 
timeout and time-in.

Sessions were divided into five blocks. In each block, the three components were presented in a random order with one constraint: If a block ended with a timeout component, the next block could not begin with a timeout component. Each presentation of the VI components lasted until six reinforcers had been delivered; because of the VI 30-s schedule, the mean component duration was programmed for approximately three min. The timeout component was programmed for three min, but to facilitate extinction of responding during this period, the component did not end within one min of a response.

Discrimination training continued until responding in both VI components met the stability criteria described below (25-76 sessions) and the response rate in the timeout component was 10 percent or less of the mean of the rates in the VI components for six consecutive sessions. All rats achieved the second criterion within the first 10 sessions of the discrimination training.

\section{Experimental Conditions}

In the experiment proper, a two-component multiple schedule was in effect. The baseline component served as the basis of comparison for responding in the punishment ${ }^{1}$ component. Independent VI 30-s schedules of food reinforcement were programmed in both components. In the baseline component, the food-only lever was inserted into the chamber and its associated cue light was turned on; satisfying the VI schedule requirement produced a food reinforcer. In the punishment component, the timeout lever was inserted and its associated cue light was turned on;

\footnotetext{
${ }^{1}$ For the rest of the experiment, the term punishment, instead of timeout, will be used to describe the component in which the timeouts occur. This was done to avoid confusion when referencing the term "timeout" in relation to (a) the component in which timeouts occur and (b) the stimulus event called a timeout. Although we are assessing the degree to which the timeouts functionally punish responding, the use of the term punishment here does not imply that a punitive effect will be found.
} 
satisfying the VI 30-s requirement produced a reinforcer and satisfying the VR 5 requirement produced a 30-s timeout. During a timeout, the VI schedule was suspended but the lever remained in place, the cue light above the lever was turned off, and the white noise was replaced with a 1000-Hz tone. Responses on the lever during a timeout, although recorded, had no effect. Because the VI and VR schedules operated conjointly, a single response could satisfy both schedules simultaneously. In those cases, the response satisfied the VI schedule and produced a food pellet; the response was not counted toward the VR schedule of timeout. Each component lasted until six pellets had been delivered. The two components were alternated until each had been presented five times.

Table 1 shows the experimental conditions (in order) and the number of sessions in each. The experimental conditions differed in the programmed rate of reinforcement during time-in. The VI schedules had mean durations of 10 s, 20 s, 30 s, 60 s, and 120 s, which respectively yielded mean programmed reinforcement rates of $6,3,2,1$, and 0.5 pellets per min. In the first condition for each rat, the reinforcement rate was 2 pellets per min. The order of the next four conditions for Rats CT30, CT31, and CT32 was opposite of the order for Rats CT29 and CT33. The sixth condition was a replication of the first condition for all rats.

\section{Stability Criteria}

Each condition lasted a minimum of 20 sessions and until responding in each component was judged stable according to the following criteria: First, there could be no increasing or decreasing trend in response rates across the most recent six sessions as judged by visual inspection. Second, the difference between the mean response rate in the first three sessions and last three sessions had to be within 10 percent of the six-session mean. 
Table 1

The experimental ( 0.5 to 6 pellet deliveries per min) conditions (in order, left to right) and the number of sessions in each.

\begin{tabular}{ccccccc}
\hline & \multicolumn{7}{c}{ Programmed Deliveries per Min } \\
\cline { 2 - 7 } Rat & 2 & 6 & 3 & 1 & 0.5 & 2 \\
\hline CT30 & 29 & 25 & 20 & 37 & 27 & 37 \\
CT31 & 22 & 43 & 27 & 30 & 21 & 32 \\
CT32 & 56 & 37 & 27 & 27 & 21 & 29 \\
\hline & & & Programmed Deliveries per Min & \\
\hline Rat & 2 & 0.5 & 1 & 3 & 6 & 2 \\
\hline CT29 & 35 & 32 & 47 & 24 & 46 & 21 \\
CT33 & 26 & 40 & 24 & 32 & 38 & 40 \\
\hline
\end{tabular}




\section{Results}

Table 2 shows the obtained reinforcement rates (total number of pellets / total time spent in time-in) in relation to the programmed rates in each component, aggregated across the stable sessions of each condition. The obtained rates closely approximate the programmed rates for all conditions, providing evidence that changes to the VI schedule across conditions successfully altered the reinforcement rates. In addition, the obtained rates in the baseline (BL) components closely approximate the rates in the punishment (PUN) components for all conditions, providing evidence that neither timeout deliveries nor changes in response rates in the punishment components (described below) altered the obtained reinforcement rates.

Table 3 shows the duration of time-in in the baseline and punishment components. All values are expressed as means and standard deviations for the stable sessions of each condition. The minimum duration of time-in required to produce 30 pellets in each component is shown in the rightmost column of Table 3 . Across conditions, the minimum was determined by the VI schedule, which required that a specified interval elapsed before a response could be reinforced. Because each session ended after 60 reinforcers - 30 per component - all 60 intervals set up by the VI schedule had to elapse. The condition with 2 reinforcers per min, for example, required that at least $30 \mathrm{~min}-15 \mathrm{~min}$ per component - had to elapse before all 60 reinforcers could be obtained. The minimum duration of time-in per component for the $0.5,1,2,3$, and 6 reinforcers per min conditions were $60,30,15,10$, and 5 min, respectively. If the rat's response rate in the component was high enough to obtain each reinforcer as soon the intervals elapsed, then the duration of time-in would approximate the minimum for that condition.

The values in Table 3 show that the total duration of time-in for each component did in fact closely approximate the minimum required by the schedule, demonstrating that the 
Table 2

Obtained pellet delivery rates in relation to the programmed rates across the baseline (BL) and punishment (PUN) components for each experimental condition. The conditions are shown in order of occurrence (left to right). The rates were aggregated (total number of pellets/total time spent in time-in) across the 6 stable sessions for each condition.

\begin{tabular}{|c|c|c|c|c|c|c|c|c|}
\hline & \multicolumn{8}{|c|}{ Programmed Deliveries per Min } \\
\hline & 2 & 6 & 3 & & 1 & & .5 & 2 \\
\hline Rat & BL PUN & BL PUN & BL PUN & BL & PUN & BL & PUN & BL PUN \\
\hline CT30 & $\begin{array}{ll}1.96 & 1.91\end{array}$ & $5.65 \quad 5.42$ & $2.91 \quad 2.80$ & 0.96 & 0.97 & 0.48 & 0.48 & $\begin{array}{ll}1.89 & 1.93\end{array}$ \\
\hline CT31 & $\begin{array}{ll}1.89 & 1.84\end{array}$ & $5.41 \quad 5.38$ & $2.83 \quad 2.84$ & 0.96 & 0.95 & 0.47 & 0.47 & 1.851 .86 \\
\hline \multirow[t]{3}{*}{ CT32 } & $1.91 \quad 1.94$ & $5.67 \quad 5.40$ & $2.90 \quad 2.91$ & 0.96 & 0.97 & 0.48 & 0.48 & 1.911 .93 \\
\hline & \multicolumn{8}{|c|}{ Programmed Deliveries per Min } \\
\hline & 2 & 0.5 & 1 & & 3 & & 6 & 2 \\
\hline Rat & BL PUN & BL PUN & BL PUN & BL & PUN & $\mathrm{BL}$ & PUN & BL PUN \\
\hline CT29 & 1.941 .95 & 0.470 .48 & 0.940 .96 & 2.75 & 2.73 & 5.48 & 5.08 & 1.901 .90 \\
\hline CT33 & 1.961 .94 & $0.48 \quad 0.47$ & 0.970 .96 & 2.91 & 2.84 & 5.69 & 5.51 & 1.941 .92 \\
\hline
\end{tabular}


Table 3

The duration of time-in for the baseline and punishment components for each condition. All values are means (standard deviations in parentheses) over the last six sessions of each condition. The rightmost column shows the minimum required duration of time-in (Minimum Duration) that had to occur in each component per session. The minimum was determined by the programmed VI schedule of reinforcement.

\begin{tabular}{|c|c|c|c|c|c|c|}
\hline \multirow{4}{*}{$\begin{array}{l}\text { Rat } \\
\text { CT30 }\end{array}$} & \multirow{3}{*}{$\begin{array}{c}\begin{array}{c}\text { Pellets per } \\
\text { Min }\end{array} \\
2\end{array}$} & \multicolumn{4}{|c|}{ Time-in Duration (min) } & \multirow{3}{*}{$\begin{array}{c}\text { Minimum } \\
\text { Duration (min) } \\
15\end{array}$} \\
\hline & & \multicolumn{2}{|c|}{ Baseline } & \multicolumn{2}{|c|}{ Punishment } & \\
\hline & & 15.33 & $(0.04)$ & 15.69 & $(0.26)$ & \\
\hline & 6 & 5.31 & $(0.06)$ & 5.53 & $(0.08)$ & 5 \\
\hline & 3 & 10.30 & $(0.06)$ & 10.71 & $(0.14)$ & 10 \\
\hline & 1 & 31.27 & $(0.16)$ & 30.93 & $(0.27)$ & 30 \\
\hline & 0.5 & 62.80 & $(0.63)$ & 61.90 & $(0.41)$ & 60 \\
\hline & 2 & 15.86 & $(0.15)$ & 15.57 & $(0.19)$ & 15 \\
\hline \multirow[t]{6}{*}{ СТ31 } & 2 & 15.86 & $(0.14)$ & 16.34 & $(0.25)$ & 15 \\
\hline & 6 & 5.55 & $(0.13)$ & 5.58 & $(0.06)$ & 5 \\
\hline & 3 & 10.60 & $(0.08)$ & 10.57 & $(0.07)$ & 10 \\
\hline & 1 & 31.19 & $(0.21)$ & 31.61 & $(0.20)$ & 30 \\
\hline & 0.5 & 63.79 & $(0.37)$ & 64.29 & $(0.77)$ & 60 \\
\hline & 2 & 16.26 & $(0.33)$ & 16.10 & $(0.17)$ & 15 \\
\hline \multirow[t]{6}{*}{ CT32 } & 2 & 15.72 & $(0.10)$ & 15.47 & $(0.08)$ & 15 \\
\hline & 6 & 5.29 & $(0.02)$ & 5.55 & $(0.09)$ & 5 \\
\hline & 3 & 10.34 & $(0.08)$ & 10.33 & $(0.06)$ & 10 \\
\hline & 1 & 31.09 & $(0.21)$ & 31.01 & $(0.37)$ & 30 \\
\hline & 0.5 & 62.55 & $(0.65)$ & 62.77 & $(0.66)$ & 60 \\
\hline & 2 & 15.75 & $(0.14)$ & 15.57 & $(0.11)$ & 15 \\
\hline \multirow[t]{6}{*}{ СТ29 } & 2 & 15.47 & $(0.05)$ & 15.37 & $(0.06)$ & 15 \\
\hline & 0.5 & 63.55 & $(0.37)$ & 63.04 & $(0.76)$ & 60 \\
\hline & 1 & 31.85 & $(0.25)$ & 31.25 & $(0.12)$ & 30 \\
\hline & 3 & 10.89 & $(0.13)$ & 11.01 & $(0.24)$ & 10 \\
\hline & 6 & 5.47 & $(0.18)$ & 5.90 & $(0.14)$ & 5 \\
\hline & 2 & 15.76 & $(0.14)$ & 15.76 & $(0.11)$ & 15 \\
\hline \multirow[t]{6}{*}{ СТ33 } & 2 & 15.30 & $(0.05)$ & 15.46 & $(0.15)$ & 15 \\
\hline & 0.5 & 62.73 & $(0.58)$ & 63.80 & $(1.23)$ & 60 \\
\hline & 1 & 31.02 & $(0.27)$ & 31.22 & $(0.45)$ & 30 \\
\hline & 3 & 10.30 & $(0.05)$ & 10.58 & $(0.05)$ & 10 \\
\hline & 6 & 5.27 & $(0.05)$ & 5.45 & $(0.04)$ & 5 \\
\hline & 2 & 15.48 & $(0.11)$ & 15.62 & $(0.15)$ & 15 \\
\hline
\end{tabular}


reinforcers were obtained as soon after each interval of the VI schedule had elapsed. Notably, there were no meaningful differences between the baseline and punishment components for any of the conditions; reductions in response rate within the punishment components (described below) did not prevent the timely delivery of reinforcers.

The left column of Figure 1 shows response rates (lever presses per min in time-in) as a function of the pellet delivery rate for each rat. The results are the means $( \pm 1$ SD) for each component, averaged over the stable sessions in each condition. Response rates in each condition were calculated separately for the baseline (black circles) and punishment (white circles) components. The black and white triangles at 2 pellets per min show the replication of this condition. Across conditions, response rates in the baseline and punishment components generally increased as the pellet delivery rate increased. This direct relation was observed regardless of the order conditions (see Table 1 for the order for each rat). For all rats, response rates peaked in the richer conditions (2-6 pellets per min); raising the pellet delivery rate after this point did not appear to affect response rates for four of the five rats.

Response rates in the baseline and punishment components diverged in the richer conditions for all rats, with lower rates in the punishment component. The rates diverged at 2 pellets per min for three rats (CT30, CT31, CT33), 3 pellets per min for rat CT32, and 6 pellets per min for rat CT29. In six of the 10 replicated components, the second set of rates was close to the first set; in the other four replications, either the baseline rate was lower (CT30), the punishment rate was lower (CT29), or both rates were lower (CT33). In each of these cases, the difference between the initial and replicated rates was small, such that the direct relation between the pellet delivery rate and response rate was preserved. Overall, the results show that the timeouts punished responding in the richer conditions. 

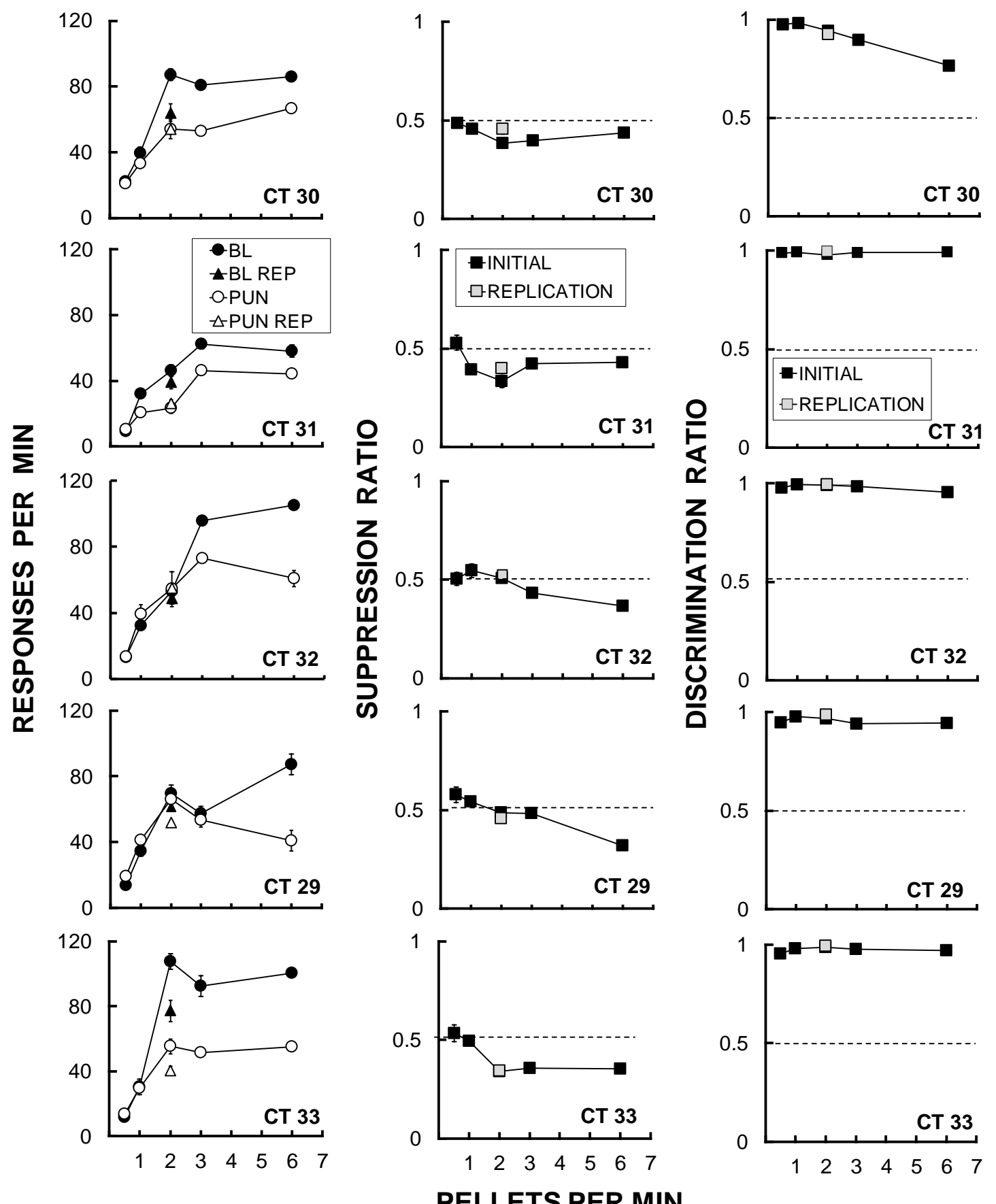

Figure 1. Mean response rates during the baseline (BL) and punishment (PUN) components (left column), mean suppression ratios (middle column), and mean discrimination ratios (right column) as a function of the pellet delivery rate. All error bars extend 1 SD above and below the mean; in some cases, the error bars are not visible because the data point overlaps them entirely. In some cases, the data points from the replications overlap the initial condition entirely (grey squares). Results are from the last six sessions of each condition. 
Table 4 shows the timeout rate (timeouts per min of time-in), total number of timeouts, and duration of the timeouts per session. All values are expressed as means and standard deviations for the stable sessions of each condition. The rate of timeout production was higher in the richer conditions for all rats. As the richer conditions produced higher response rates (shown in the left column of Figure 1), the VR-5 schedule of timeout necessarily led to more frequent timeouts.

The table shows that the total absolute number of timeouts and the amount of the session spent in timeout were lower in the richer conditions. This can be attributed to the reduced opportunity to produce timeouts. The opportunity to produce timeouts is dependent on the duration of time-in during the punishment component. As shown in punishment component column of Table 3, the time spent in time-in was inversely related to the reinforcement rate. In the richest condition, 6 pellets per min, the rats spent less than six minutes in time-in (range: 5.45 - $5.90 \mathrm{~min}$ ); conversely, in the leanest condition, 0.5 pellets per min, the rats spent more than 60 min in time-in (range: 61.90 - 64.29). Therefore, the absolute number of timeouts and time spent in timeout was necessarily lower in richer conditions.

The difference in responding across the baseline and punishment components can be summarized by the suppression ratio, a measure that expresses responding in the punishment component relative to the baseline component. The middle column of Figure 1 shows suppression ratios as a function of the programmed reinforcement rate for each rat. For each of the stable six sessions of each condition, the response rate in the punishment component was 
Table 4

Timeout rate (timeouts per min), total timeouts, and timeout duration (min). All values are means (standard deviations in parentheses) over the last six sessions of each condition.

\begin{tabular}{|c|c|c|c|c|c|c|c|}
\hline \multirow{2}{*}{$\begin{array}{c}\text { Rat } \\
\text { CT30 }\end{array}$} & \multirow{2}{*}{$\begin{array}{c}\begin{array}{c}\text { Pellets per } \\
\text { Min }\end{array} \\
2\end{array}$} & \multicolumn{2}{|c|}{ Timeouts per Min } & \multicolumn{2}{|c|}{ Total Timeouts } & \multicolumn{2}{|c|}{$\begin{array}{c}\text { Timeout Duration } \\
(\mathrm{min})\end{array}$} \\
\hline & & 10.70 & $(0.49)$ & 167.83 & $(5.15)$ & 83.95 & $(2.58)$ \\
\hline & 6 & 13.14 & $(0.43)$ & 72.67 & $(2.21)$ & 36.35 & (1.11) \\
\hline & 3 & 10.45 & $(0.35)$ & 112.00 & $(4.47)$ & 56.03 & $(2.24)$ \\
\hline & 1 & 6.58 & $(0.49)$ & 203.50 & $(14.00)$ & 101.80 & $(7.00)$ \\
\hline & 0.5 & 4.15 & $(0.30)$ & 257.00 & (18.50) & 128.55 & $(9.26)$ \\
\hline & 2 & 10.65 & $(1.15)$ & 165.67 & (16.28) & 82.87 & $(8.14)$ \\
\hline \multirow[t]{6}{*}{ СТ31 } & 2 & 4.59 & $(0.66)$ & 74.83 & $(9.82)$ & 37.44 & $(4.91)$ \\
\hline & 6 & 8.39 & $(0.49)$ & 46.83 & $(2.61)$ & 23.43 & $(1.30)$ \\
\hline & 3 & 9.07 & $(0.43)$ & 95.83 & $(4.95)$ & 49.61 & $(4.32)$ \\
\hline & 1 & 4.09 & $(0.23)$ & 129.17 & (7.06) & 64.61 & $(3.53)$ \\
\hline & 0.5 & 2.04 & $(0.32)$ & 130.67 & (19.34) & 65.36 & (9.67) \\
\hline & 2 & 5.14 & $(0.44)$ & 82.67 & $(7.04)$ & 41.35 & $(3.52)$ \\
\hline \multirow[t]{6}{*}{ СТ32 } & 2 & 10.78 & $(0.60)$ & 166.67 & (9.14) & 83.38 & $(4.57)$ \\
\hline & 6 & 11.78 & $(0.82)$ & 65.33 & $(4.27)$ & 32.69 & $(2.14)$ \\
\hline & 3 & 14.45 & $(0.48)$ & 149.17 & (4.14) & 74.63 & (2.07) \\
\hline & 1 & 7.79 & (1.09) & 241.33 & (31.15) & 120.73 & (15.59) \\
\hline & 0.5 & 2.66 & $(0.37)$ & 173.00 & (26.83) & 83.46 & (10.97) \\
\hline & 2 & 10.92 & $(1.90)$ & 169.83 & $(28.79)$ & 84.96 & (14.40) \\
\hline \multirow[t]{6}{*}{ CT29 } & 2 & 13.06 & $(0.93)$ & 200.83 & (14.23) & 100.47 & $(7.11)$ \\
\hline & 0.5 & 3.81 & $(0.64)$ & 240.00 & (37.94) & 120.07 & (18.98) \\
\hline & 1 & 8.20 & $(0.29)$ & 256.33 & $(9.18)$ & 128.24 & $(4.59)$ \\
\hline & 3 & 10.46 & $(0.50)$ & 115.00 & $(4.20)$ & 57.53 & $(2.10)$ \\
\hline & 6 & 7.94 & $(0.54)$ & 46.83 & $(2.67)$ & 23.43 & (1.34) \\
\hline & 2 & 10.26 & $(0.71)$ & 161.67 & (10.69) & 80.88 & $(5.35)$ \\
\hline \multirow[t]{6}{*}{ CT33 } & 2 & 10.93 & $(0.96)$ & 168.83 & (13.46) & 84.45 & (6.74) \\
\hline & 0.5 & 2.72 & $(0.47)$ & 173.33 & $(28.73)$ & 86.71 & (14.37) \\
\hline & 1 & 5.87 & $(0.47)$ & 183.17 & (14.11) & 91.62 & $(7.06)$ \\
\hline & 3 & 10.08 & $(0.35)$ & 106.67 & $(3.40)$ & 53.35 & $(1.70)$ \\
\hline & 6 & 10.78 & $(0.66)$ & 58.67 & $(3.45)$ & 29.35 & $(1.72)$ \\
\hline & 2 & 7.89 & $(0.69)$ & 123.17 & $(9.91)$ & 61.61 & $(4.96)$ \\
\hline
\end{tabular}


divided by the sum of the response rates in the punishment component and the baseline component, yielding six suppression ratios. The middle column of Figure 1 shows the mean $( \pm 1$ $\mathrm{SD}$ ) of these ratios. Suppression ratio values below 0.5 (the dotted line on each individual graph) indicate that responding in the punishment component was lower than responding in the baseline component - a punitive effect of timeouts.

In 22 of the 30 conditions (73\%), the suppression ratios were below 0.5. Across conditions, suppression ratios decreased as the pellet delivery rate was raised for three of the five rats (CT32, СТ29, СT33). For the other two rats (СT30, СT31), no clear relation can be discerned. Close replication of the suppression ratios (shown with grey squares) occurred for all rats. These replications show that although absolute rates of responding in the baseline and punishment components did not replicate perfectly in all cases (left column of Figure 1), the proportional differences between them were preserved.

In the leaner conditions (0.5-1 pellet per $\mathrm{min})$ timeout did not suppress responding, and in some cases, it facilitated responding (suppression ratios $>0.5$ ). These facilitative effects are small, however, and must be considered alongside the low response rates engendered by leaner conditions. When response rates are low, small absolute differences between the response rates in the two components can have a large effect on the suppression ratio values. For example, consider the low response rates for CT31 in the 0.5 pellets per min condition (left column of Figure 1). Even though the data points overlap, the response rate in the punishment component is slightly higher (10.29) than the rate in baseline component (9.02), which yielded a suppression ratio of 0.53 (apparently a facilitative effect). Therefore, when absolute response rates are low, even minor differences can have a big effect on the suppression ratio.

The rats' discrimination of timeout from time-in was maintained at high levels 
throughout the experiment. Discrimination ratios for each session were calculated by dividing the response rate during the time-in portion of the punishment component by the sum of the rates in time-in and timeout. The right column of Figure 1 shows the mean ratios $( \pm 1 \mathrm{SD}$; overlapped by the data point in all cases) for the stable sessions in each condition. Discrimination was nearly perfect (range: $0.9-1.0$ ) in 29 of the 30 conditions $(97 \%)$, showing that the suppression in response rates cannot be attributed to changes in the discriminability of time-in and timeout across conditions.

The analyses in Figure 1 summarize the overall effects of timeout on responding. Additional analyses were conducted to assess the short-term effects of the timeouts. The left column of Figure 2 shows the median time (and interquartile ranges) between the end of a timeout and the resumption of responding. For all rats, post-timeout response latencies decreased as a function of reinforcement rate. In other words, responding after a timeout resumed more rapidly as the reinforcement rate was enriched. This is in contrast with the suppression ratio analysis (middle column of Figure 1), which showed that overall responding was lower in the richer conditions. The latency analysis demonstrates that the suppression found in the richer conditions cannot be attributed to a short-term disruption of responding by the timeouts. The right column of Figure 2 shows the median time (and interquartile ranges) between the end of a reinforcer and the resumption of responding in the baseline and punishment components. Postreinforcer latencies also decreased as a function of reinforcement rate. Consistent with previous literature on interval schedules (Catania \& Reynolds, 1968), response latencies were inversely related to the pellet delivery rates. Overall, there were no systematic differences in the latency to respond after a timeout or a reinforcer. It appears that both types of latencies were controlled by the reinforcement rate. 

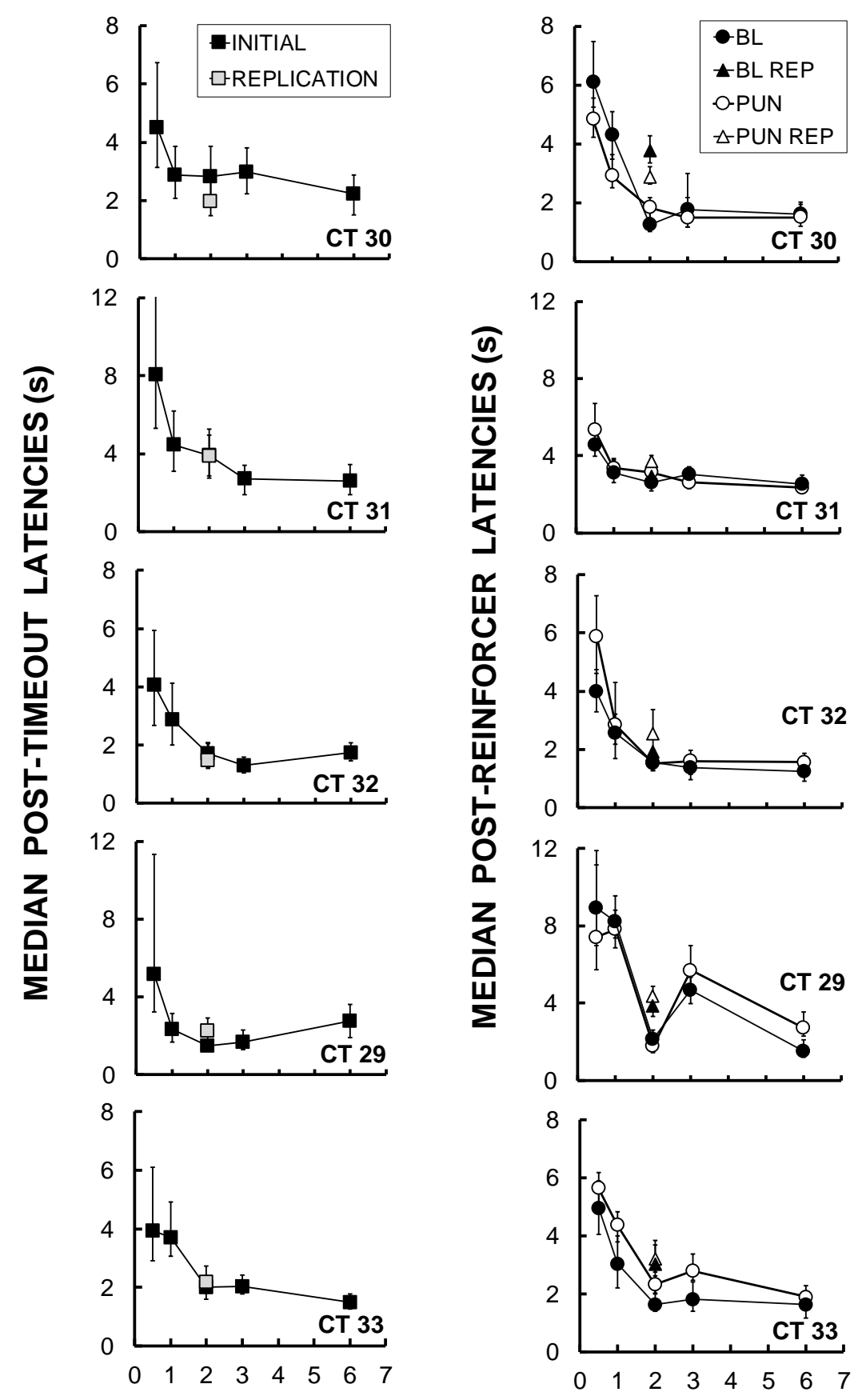

\section{PELLETS PER MIN}

Figure 2. Median post-timeout latencies (s) as a function of the pellet delivery rate (left column) and median post-reinforcer latencies (s) during the baseline (BL) and punishment (PUN) components as a function of the pellet delivery rate (middle column). All error bars extend from the $25^{\text {th }}$ to $75^{\text {th }}$ percentiles. In some cases, the data points from the replications overlap the initial condition entirely. 
When two or more timeouts are delivered in the absence of a reinforcer, there are extended stretches of time with no reinforcer deliveries. It is possible that the observed reductions in responding are not the result of punishment by the timeouts, but instead simply reflect a progressive weakening of responding as the stretch of time without a reinforcer increases. Evidence of this weakening would be an increase in response latency as a function of consecutive timeouts. Figure 3 shows median latencies as a function of consecutive timeouts. The different symbols signify the different reinforcement rates $(0.5-6$ pellets per min $)$. Linear functions were fit to each set of response latencies in the different conditions and the explained variance values $\left(\mathrm{r}^{2}\right)$ are displayed in the lower right-hand panel of the figure. Linear functions with a positive slope would provide evidence of increased latencies as a function of consecutive timeouts. However, only three of the $25(12 \%)$ linear functions had a positive slope equal to or greater than 0.1 (range: $0.1-0.28$ ). Instead, the majority of the linear functions (18 out of 25 , $72 \%$ ) had a negligible slope and there were no good fits to the data (when the slope of the line is close to zero, it is expected that the fit of the line would be low, as shown by the $\mathrm{r}^{2}$ values). Overall, the figure shows that there were no systematic changes in post-timeout latencies as a function of consecutive timeouts for any of the conditions. Although consecutive timeouts extend the time since the last reinforcer - by up to 20 minutes in the most extreme cases - there was no evidence that responding was weakened by this aspect of the experimental environment.

Figure 3 is consistent with the analysis in the left column of Figure 2; the post-timeout latencies were higher for the lean conditions and lower for rich conditions. The latencies in the leanest condition, 0.5 pellets per min (white circles), showed the most marked difference from the other conditions for all rats. The second leanest condition, 1 pellet per min (black triangles), was only markedly higher than the three richer conditions for two rats (CT32, CT33). The 


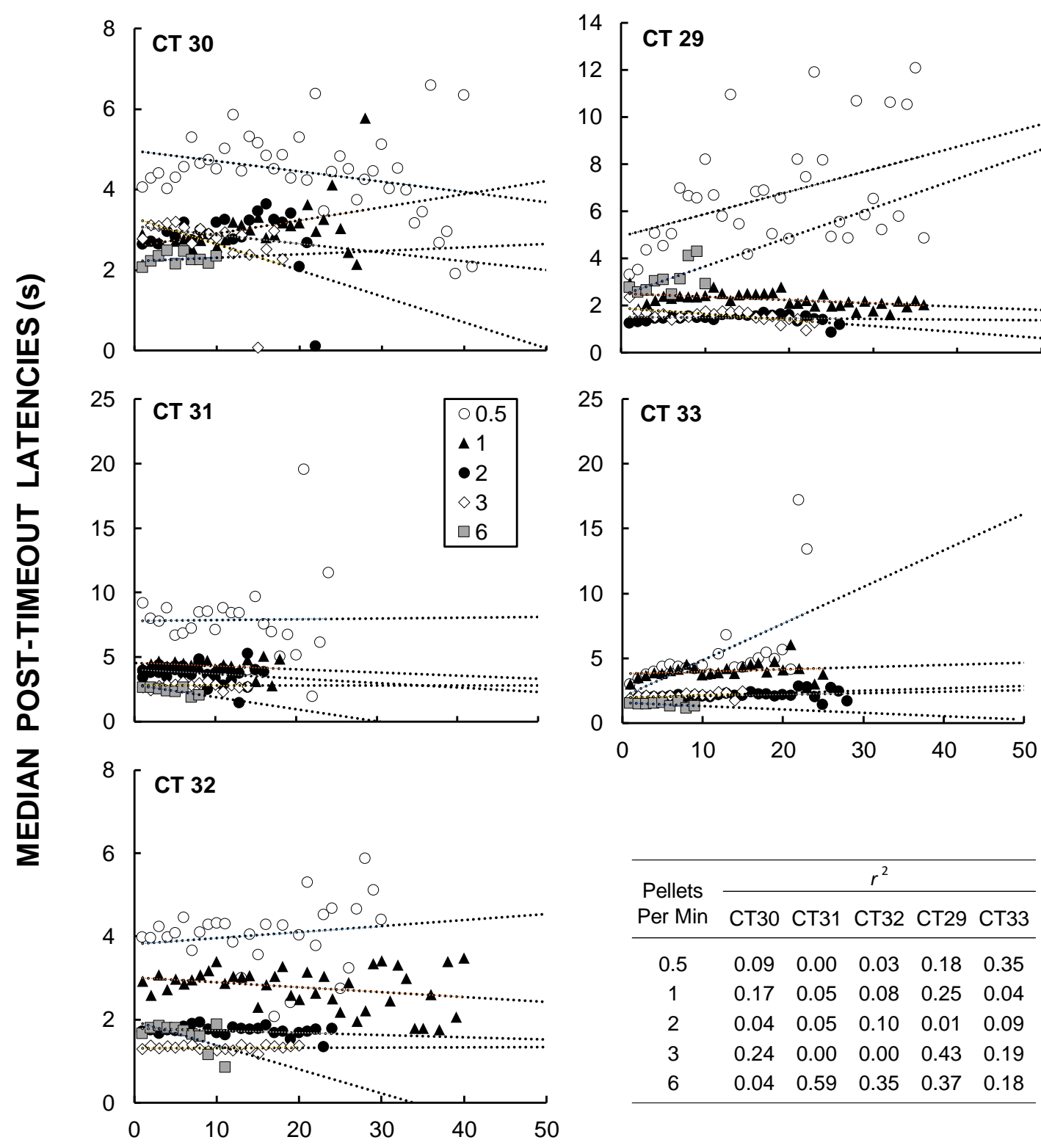

CONSECUTIVE TIMEOUTS

Figure 3. Median post-timeout latencies (s) as a function of consecutive timeouts. Timeouts are considered consecutive when no reinforcer occurs between the offset of one timeout and the onset of the next timeout. The panel in the lower right-hand corner shows the goodness of fit for each of the lines fitted to the results from each condition. 
differences in the latencies for the richer conditions were less discernable, as evidenced by the overlap in the data points for the 2 (black circles), 3 (white diamonds), and the 6 (grey squares) pellets per min conditions. As in the left column of Figure 2, the response latencies following timeouts are controlled by the pellet delivery rate. Figure 3 expands this analysis by showing that the pellet delivery rate continues to control the response latencies even when the pellets have not been delivered for extended periods (following consecutive timeouts).

Finally, Figure 3 shows that the number of consecutive timeouts that occurred was inversely related to the reinforcement rate. For four of the five rats, the number of consecutive timeouts that occurred was high in the two leanest conditions ( 0.5 and 1 pellets per min), moderate in two of the richer conditions ( 2 and 3 pellets per min), and low in the richest condition (6 pellets per min). As with the total number of timeouts per session in Table 4 , this can be attributed to the reduced time spent in time-in - and thus reduced opportunity - to produce timeouts as the reinforcement rate was enriched.

\section{Discussion}

This experiment was concerned with the role of reinforcement rate during time-in on the aversiveness of timeout. Whereas previous basic research has assessed aversiveness by studying behavior that avoids timeout, the present experiment studied behavior that is punished by timeout. The results confirm that the reinforcement rate during time-in is important: When reinforcement rates were relatively low, timeout failed to punish the rats' lever pressing. At sufficiently high rates, timeout was an effective punisher. The point at which the timeout became effective differed across rats, from 1 pellet per min (via a VI 60-s schedule) to 4 pellets per min (via a VI 15-s schedule). 
Fantino's (1977) delay-reduction hypothesis predicted that the aversiveness of timeouts should be directly related to the reinforcement rates in time-in. For three of five rats (CT29, CT32, CT33), raising the reinforcement rate did lead to progressive increases in the punitive effect of timeout (as indicated by decreasing suppression ratios; see Figure 1). For the other two rats (CT30, CT31), raising the reinforcement rate only led to progressive increases up to 2 pellets per min. Support for Fantino's hypothesis may be limited in some cases when the reinforcement rate becomes sufficiently rich.

The following sections will explore the present findings in relation to the relevant literature. First, the results will be discussed in relation to results from avoidance experiments and a model describing how the aversiveness of timeout may exert effects in a punishment paradigm will be presented. Next, experiments designed to assess the role of reinforcement rate on the punitive function of a different aversive stimulus, electric shock, will be described. Finally, the potential clinical significance of the present methods and primary findings will be addressed.

\section{Analyzing Aversiveness of Timeout}

Timeout's aversiveness is evaluated by assessing changes in behavior in either avoidance or punishment experiments (Leitenberg, 1965). Although reinforcement rate has been shown to be a controlling variable of timeout's aversiveness in avoidance experiments (Holtyn, 2012; Richardson \& Baron, 2008), timeout's aversiveness in punishment experiments has not been sufficiently explored. Because of this lack of research, it has been difficult to assess whether reinforcement rate interacts with timeout aversiveness in similar ways across paradigms. In the following two sections, a comparison of reinforcement rate's role in timeout aversiveness across avoidance and punishment experiments will be addressed. 
Range of aversive effects. An interesting difference between the present results and the results from timeout avoidance experiments can be seen in the range of timeout's aversive effects. In Holtyn's (2012) study, rats could respond to avoid upcoming timeouts from responseindependent food deliveries. The rate of the response-independent food deliveries was manipulated across conditions. The rats engaged in the lowest avoidance rates during the leanest condition ( 0.125 food deliveries per min) and therefore, received most of the programmed timeouts. As the food delivery rate was raised across conditions, avoidance rates and the percentage of timeouts avoided systematically increased to the point that most of the rats avoided nearly all of the timeouts in the richest condition ( 2 food deliveries per min). The clear functional relations obtained in Holtyn's (2012) avoidance study spanned the entire range of potential aversive effects from no avoidance of timeouts to avoidance of all timeouts. By comparison with Holyn's (2012) study, the present experiment produced a smaller range of aversive effects, ranging from no punishment of responding to a punitive effect great enough to reduce responding by half. There were no cases in which timeouts reduced responding to zero or nearzero levels, an effect that could be considered a parallel to the successful avoidance of all timeouts in an avoidance study.

An argument might be made that the timeouts in the present experiment might have suppressed responding further if the reinforcement rate had been raised further. This seems unlikely, in light of the results from the three rats that did not show graded suppression in conditions with reinforcement rates above 2 pellets per min. For these rats, response rate increases appear to reach an asymptote (sometimes referred to as a "locked rate") in richer conditions, demonstrating the established effect of further raises to the reinforcement rate on VI schedules (Catania \& Reynolds, 1968). Catania and Reynolds (1968) showed that individual 
subject's responding on VI schedules generally reaches the characteristic asymptote at different levels. Therefore, it seems likely that the additional two rats would reach this point if the reinforcement rate had been raised further. In conclusion, it is unlikely that the failure to reduce response rates below half was due to the spread of reinforcement rate parameters chosen in the present experiment.

The present punishment experiment can be conceptualized as an alternative method to measure the aversiveness of timeout. Although the direction of the functional relation between reinforcement rate and timeout's aversive effects was consistent with Holtyn's (2012) avoidance study, a full range of aversive effects was not observed in the present punishment experiment. It could be argued that these differences suggest that reinforcement rate may be less of a contributing factor to timeout's aversiveness in a punishment experiment. Although this may be a reasonable argument, an alternative explanation of reinforcement rate's contributions is possible.

A punishment model of timeout's aversiveness. A large body of evidence already exists that suggests that timeouts from positive reinforcement can be aversive when the timeouts are used as punishers (e.g., see reviews by Azrin \& Holz, 1966; Everett et al., 2010; Lerman \& Vorndran, 2002). In the present experiment, the assumption was made that the punitive effects of timeout - shown by reductions in the response rate - would be an accurate reflection of timeout's aversiveness. Simply put, the punitive effects of timeout were expected to change in concert with the aversiveness of timeout. It is possible, however that the punitive and aversive effects of timeout are not aligned.

Consider the contributions of the consequences in a punishment paradigm. At any given time, the current response rate reflects the net effect of the contingencies associated with the 
reinforcement and timeout deliveries. Reinforcement strengthened the lever press (responding was maintained in both components). At the same time, the contingent timeouts weakened the lever press (response reductions in the punishment component). Thus, the overall response rate in any given punishment component was a manifestation of these combined - and counteracting - contingencies.

Because the relative strengthening and weakening effects are unknown, the punitive effects could take several forms. Consider the three hypothetical functions in Figure 4. The xaxis represents reinforcement rate and the y-axis represents response strength. The rising dotted line represents the effects of increasing reinforcement on response strength. The direction of reinforcement's strengthening effects are well known - as the reinforcement rate is raised, the response rate function is monotonically increasing and negatively accelerated (Catania \& Reynolds, 1958, Herrnstein, 1970). This line remains the same across the three graphs. The timeout avoidance experiments (Holtyn, 2012; Richardson \& Baron, 2008) and Fantino's (1977) hypothesis suggest that timeout should become increasingly aversive as the reinforcement rate is raised. Therefore, the falling dashed line represents the increasing aversiveness of the timeouts on response strength. If the aversive effects of timeout are equal and opposite to the strengthening effects of the reinforcement rate - shown by the leftmost graph - then there would be no change to the net response strength. Therefore, the punitive effect of timeout would remain unchanged when the reinforcement rate is manipulated. If the aversive effects contribute less than the effects of reinforcement rates - shown by the middle graph - then the punitive effect of the timeouts would get weaker when the reinforcement rate is raised. Finally, if the aversive effects contribute more than the effects of reinforcement rate - shown by the rightmost graph - 

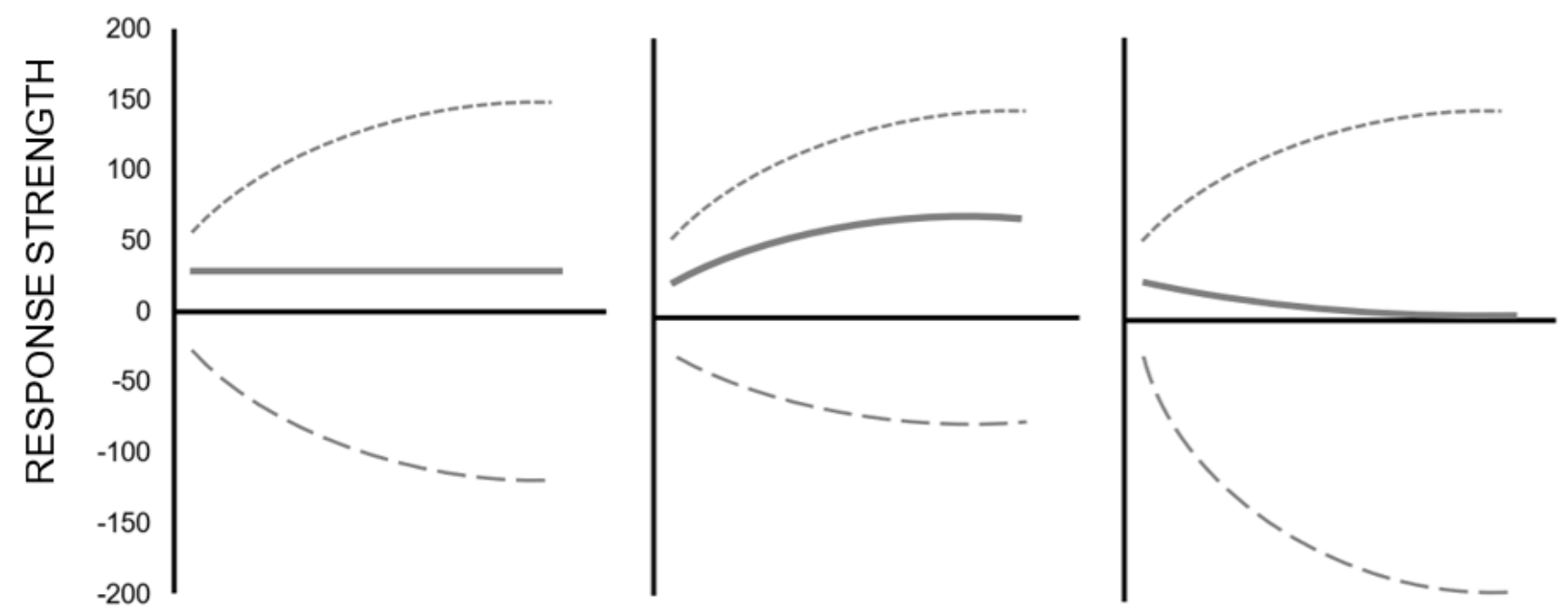

REINFORCEMENT RATE

$$
\begin{array}{ll}
--------\cdot & \text { FOOD } \\
----- & \text { TIMEOUT } \\
& \text { PUNITIVE EFFECTS }
\end{array}
$$

Figure 4. Response strength as a function of reinforcement rate. The dotted line represents the contributions of reinforcement to response strength. The dashed line represents the contributions of timeouts to response strength. The solid line represents the net effect of both reinforcement and timeout on response strength. 
then the punitive effect of the timeouts would get stronger when the reinforcement rate is raised.

Notice that in all of the hypothetical cases on Figure 4, the aversiveness of the timeouts is in fact related to the reinforcement rate: when the reinforcement rate is raised, timeout becomes more aversive. Despite this, it is possible to obtain relations between reinforcement rate and the punitive effect of timeout that differ in direction. Under this model, the punitive effects of the timeout may not reflect the aversiveness of timeout.

The interpretive problems described above for the present punishment experiment are not present in avoidance studies. In avoidance studies, the food deliveries are response-independent. The time-in period is enriched by raising the rate at which the response-independent food is delivered. This enrichment of the time-in periods is independent of any strengthening or weakening effects of the avoidance response. That is, there is no competition between the strengthening effects of food and the aversiveness of timeouts because the food deliveries do not strengthen the response. Therefore, any changes in the avoidance response rate can be attributed to changes in the aversiveness of the timeout. Holtyn's (2012) results showed clearly that when time-in was enriched systematically, the timeouts became increasingly aversive, which engendered systematic increases in the avoidance response.

In summary, experimental studies on the aversiveness of timeout have adopted substantially different experimental strategies. The differences in the contingencies present in each of these strategies appears to contribute to the differences in the results. Basic studies on the aversiveness of timeout are likely to yield more readily interpretable results if an avoidance paradigm - instead of a punishment paradigm - is used.

\section{Comparison with Shock Punishment}

Just as the experimental strategy is important, so too are the stimuli used as the 
reinforcers and punishers. In the present experiment, the punisher was located on the same dimension as the reinforcer. The reinforcer for the response was the delivery of food and the punisher for the response was a discriminable period of time in which food was absent. The punishers were necessarily linked to the reinforcers and the reinforcement rate. It is possible to arrange punishing stimuli that fall on a separate dimension than the reinforcer. A commonly used punishing stimulus in basic research is electric shock, which is completely unrelated to the reinforcer.

Previous research on the role of reinforcement rate in shock punishment has yielded discrepant findings. Only three studies on shock punishment that manipulated the reinforcement rate could be found. Each of these studies will be discussed. In the first study, conducted by Church and Raymond (1967), rats' lever pressing was punished by shock deliveries (0.15 mA, 2 s duration). For one group of rats, the response was maintained on a VI schedule with a lean reinforcement rate of 0.2 pellets per min. In the other group, the VI schedule arranged a rich reinforcement rate of 5 pellets per min. In the baseline sessions, the mean response rates for the rats under the lean schedule were much lower than the mean rates for the rats under the rich schedule (10 vs. 30 responses per min). During punishment sessions, half of the rats from each group received shocks on a VI 2-min schedule. Mean response rates in the lean group were reduced to 2 responses per min, an 80 percent reduction. Response rates in the rich group were reduced to 23 responses per min, a 23 percent reduction. The shocks were more punitive when the schedule of reinforcement was lean.

In the second study, Bouzas (1978) studied the relation between shock and reinforcement rate using a two-component multiple schedule. In one component, pigeons' key pecking was maintained on a VI schedule that arranged a rich reinforcement rate of (1 grain presentation per 
min). In the other component, responding was maintained on a VI schedule that arranged a lean reinforcement rate ( 0.25 grain presentations per min). Following a baseline condition, shocks were presented on a VI 30-s schedule of punishment in both components. Across the rest of the conditions, the intensity of the shock was manipulated from $1 \mathrm{~mA}$ to $6 \mathrm{~mA}$. In the baseline condition, the mean response rates were higher on the rich schedule than the lean schedule for all pigeons. As the shock intensity was increased across conditions, response rates gradually decreased in both components for all pigeons. A proportion of baseline measure (punished response rate / baseline response rate) was calculated for each component across conditions. As the shock intensity increased across conditions, greater proportional reductions were always evident in the component with the lean schedule. As in Church and Raymond's (1967) study, the shocks were more punitive when the schedule of reinforcement was lean.

In the third study, Holz (1968) studied the relation between shock and reinforcement rate using a concurrent schedule with two response options. Pigeons responded on two keys that were available simultaneously and produced food reinforcers according to two independent VI schedules. Pecks on one key were maintained on a relatively lean schedule $(0.13$ grain presentations per min). Pecks on the second key were maintained on a relatively rich schedule (0.53 grain presentations per min). After baseline sessions, every peck produced a shock. Across the rest of the conditions, the intensity of the shock was manipulated from $3 \mathrm{~mA}$ to $12 \mathrm{~mA}$. In the baseline sessions, higher response rates were maintained by the richer schedule. As the shock intensity was increased across conditions, response rates on both keys gradually decreased for all pigeons. The proportional decreases for each key across the shock intensities were always equivalent, indicating that the punitive effects of shock were independent of reinforcement rate. Consider how the shock studies would fit into the model on Figure 4. The strengthening 
of reinforcement would be expected to remain the same. The aversiveness of the shock, however, would look different. Because shock is not directly related to the reinforcer, shock's aversiveness would presumably remain constant (a straight line). With the aversiveness of shock remaining the same, the relation between the reinforcement rate and aversiveness of shock would be clear. Two of the shock studies (Bouzas, 1978; Holz (1968), however, manipulated the intensity of the shock across conditions. This manipulation would be expected to alter shock's aversiveness. As in the present experiment, the manner in which the aversiveness changes in conjunction with changes to reinforcement rate would be unknown, thereby creating similar interpretative problems for the punitive function. In Church and Raymond's study (1967), the shock parameters remained the same, which removes the interpretive problems just discussed. Under the model in Figure 4, reinforcement would strengthen responding more in the rich group than in the lean group and shock's aversiveness would remain the same. Their results were consistent with this model: responding was higher for the rich group, indicating that the increased reinforcement rate contributed more to the response rate.

The main issue with Church and Raymond's (1967) study is the small range of reinforcement rates that were evaluated. Because there were only two different reinforcement rates, no clear functional relations can be determined. Therefore, it is possible that their results only obtained a small snapshot of the relation between reinforcement rate and the effects of shock. Perhaps replications of the previous experimental arrangements with a wider range of reinforcement rates would be fruitful.

\section{Clinical Significance}

The present experiment evaluated one factor hypothesized to alter the effects of timeout from positive reinforcement, a common intervention in clinical settings. When designing an 
intervention, the clinician is tasked with choosing appropriate parameters, such as the duration of the timeout. Ideally, these parameter choices are based on empirical evidence and consider the possible contributions of other situational variables such as the ongoing reinforcement rate for the problem behavior. An understanding of how the parameters alter the aversiveness of timeout may lead to more effective interventions in clinical settings.

Although the primary findings support the relevance of the ongoing reinforcement rate for timeout's effectiveness, the present experiment was not designed as an analog to the clinical situation. During timeouts in the present experiment, there was only one response option and only one programmed source of reinforcement. This design set up a sizably different situation than is commonly seen in clinical settings and may be seen as a limitation to the clinical significance of the results. Consider a classic example of how timeouts are implemented in general education classrooms. When a student misbehaves, the teacher sends the student out of classroom - often into the hallway - for the duration of the timeout. In this example, the opportunity to obtain reinforcement for the misbehavior is not all that has been removed. Instead, an array of potential responses and reinforcers has been removed. It is possible that timeout punishment is more effective in clinical settings than in the present experiment because the timeouts remove more than just the reinforcer for the target response.

Future basic research should consider how timeout could be enhanced by altering the number of alternative responses to obtain the reinforcer that maintains the target response. For example, if teacher attention maintains the problem behavior, the student could be taught to engage in alternative, appropriate behavior to recruit teacher attention. One way to achieve an analogous experimental arrangement is to provide multiple responses options. One response would be the target response and the other responses would be 'alternative' responses. All three 
of the responses would be maintained by independent schedules of reinforcement. Without timeouts, responding would be allocated across the options according to the programmed reinforcement rate on each response option. In timeout conditions, instances of the target response would produce timeouts that would functionally remove the availability of reinforcement for all three responses. In this arrangement, responding is expected to shift to the 'appropriate' alternatives, thereby effectively punishing the target response.

In this example, the outcome of changes to the reinforcement rate for the multiple options could then be evaluated. One potential manipulation would be to change the reinforcement rate for all of the options simultaneously. It is possible that low reinforcement rates across the response options would lead to less suppression than high reinforcement rates. An additional manipulation would be to change the reinforcement rate for the different response options. It is possible that higher reinforcement rates for the alternative responses relative to the target response would lead to greater suppression.

Another possibility for future basic research is to examine how timeouts interact with different aspects of reinforcement, such as immediacy, in the time-in environment. In the present experiment, timeouts were delivered following the completion of the ratio requirement, regardless of whether reinforcement for the response had been obtained. This arrangement may not be fully representative of the events that occur in the clinical setting. It may be the case that the reinforcer for the problem behavior is always delivered immediately after the response, such as when the reinforcer is peer attention. In this case, the reinforcer is delivered immediately and the timeout then follows. It may be that reinforcer immediacy or probability determines the effectiveness of the timeouts. Future basic research should consider the interactions of these parameters when designing experiments on timeout. 


\section{References}

Azrin, N. H. (1960). Effects of punishment intensity during variable-interval reinforcement. Journal of the Experimental Analysis of Behavior, 3, 123-142.

Azrin, N. H., \& Holz, W. C. (1966). Punishment. In W. K. Honig (Ed.), Operant behavior: Areas of research and application (pp. 380-447). New York: Appleton-Century-Crofts.

Azrin, N. H., Holz, W. C., \& Hake, D. F. (1963). Fixed-ratio punishment. Journal of the Experimental Analysis of Behavior, 6(2), 141-148.

Beavers, G. A., Iwata, B. A., \& Lerman, D. C. (2013). Thirty years of research on the functional analysis of problem behavior. Journal of Applied Behavior Analysis, 46(1), 1-21.

Benjamin, R., Mazzarins, H., \& Kupfersmid, J. (1983). The effect of time-out (TO) duration on assaultiveness in psychiatrically hospitalized children. Aggressive Behavior, 9(1), 21-27.

Bouzas, A. (1978). The relative law of effect: Effects of shock intensity on response strength in multiple schedules. Journal of the Experimental Analysis of Behavior, 30(3), 307-314.

Brantner, J. P., \& Doherty, M. A. (1983). A review of timeout: A conceptual and methodological analysis. The effects of punishment on human behavior, 87-132.

Catania, A. C., \& Reynolds, G. S. (1968). A quantitative analysis of the responding maintained by interval schedules of reinforcement. Journal of the experimental analysis of behavior, 11(3S2), 327-383.

Church, R. M., \& Raymond, G. A. (1967). Influence of the schedule of positive reinforcement on punished behavior. Journal of Comparative and Physiological Psychology, 63(2), 329.

Crespi, T. D. (1988). Effectiveness of time-out: A comparison of psychiatric, correctional and day-treatment programs. Adolescence, 23(92), 805.

Donaldson, J. M. (2011). Evaluations of time-out parameters with young children (Doctoral 
dissertation, University of Florida).

Donaldson, J. M., \& Vollmer, T. R. (2012). A procedure for thinning the schedule of time-out. Journal of Applied Behavior Analysis, 45(3), 625-630. doi: 10.1901/jaba.2012.45-625

Everett, G. E., Hupp, S. D., \& Olmi, D. J. (2010). Time-out with parents: A descriptive analysis of 30 years of research. Education and Treatment of Children, 33(2), 235-259.

Fabiano, G.A., Pelham Jr, W.E., Manos, M.J., Gnagy, E.M., Chronis, A.M., Onyango, A.N., Lopez-Williams, A., Burrows-MacLean, L., Coles, E.K., Meichenbaum, D.L. and Caserta, D.A., 2004. An evaluation of three time-out procedures for children with attention-deficit/hyperactivity disorder. Behavior Therapy, 35(3), pp.449-469.

Fantino, E. (1977). Conditioned reinforcement: Choice and information. Handbook of operant behavior, 313-339.

Fleshler, M., \& Hoffman, H. S. (1962). A progression for generating variable-interval schedules. Journal of the Experimental Analysis of Behavior, 5, 529-530. doi: 10.1901/jeab.1962.5529

Hackenberg, T. D., \& Defulio, A. (2007). Timeout from reinforcement: Restoring a balance between analysis and application. Mexican Journal of Behavior Analysis, 33, 37-44.

Herrnstein, R.J. (1970). On the law of effect. Journal of the Experimental Analysis of Behavior, 13, 243-266. doi: 10.1901/jeab.1970.13-243.

Holtyn, A. F. (2012). Avoidance of timeout from response-independent schedules of food or sucrose water with and without a limited hold on consumption. West Virginia University Libraries.

Holz, W. C. (1968). Punishment and rate of positive reinforcement. Journal of the Experimental Analysis of Behavior, 11(3), 285-292. 
Jones, A. M. (2015). Use of timeout procedures: A national survey of educators' utilization and perceptions of the effectiveness of timeout. The University of Utah.

Kaufman, A., \& Baron, A. (1968). Suppression of behavior by timeout punishment when suppression results in loss of positive reinforcement. Journal of the Experimental Analysis of Behavior, 11(5), 595-607. doi:10.1901/jeab.1968.11-595

Larzelere, R. E., Schneider, W. N., Larson, D. B., \& Pike, P. L. (1996). The effects of discipline responses in delaying toddler misbehavior recurrences. Child \& Family Behavior Therapy, 18(3), 35-57.

Leitenberg, H. (1965). Is time-out from positive reinforcement an aversive event? A review of the experimental evidence. Psychological Bulletin, 64(6), 428. doi:10.1037/h0022657

Lerman, D. C. \& Vorndran, C. M. (2002). On the status of knowledge for using punishment: Implications for treating behavior disorders. Journal of Applied Behavior Analysis, 35, 431-464. doi:10.1901/jaba.2002.35-431

Lydon, S., Healy, O., Moran, L., \& Foody, C. (2015). A quantitative examination of punishment research. Research in developmental disabilities, 36, 470-484. doi:10.1016/j.ridd.2014.10.036

Mathews, J. R., Friman, P. C., Barone, V. J., Ross, L. V., \& Christophersen, E. R. (1987). Decreasing dangerous infant behaviors through parent instruction. Journal of Applied Behavior Analysis, 20(2), 165-169.

Michael, J. (1982). Distinguishing between discriminative and motivational functions of stimuli. Journal of the Experimental Analysis of Behavior, 37(1), 149-155. doi:10.1901/jeab.1982.37-149

Porterfield, J. K., Herbert-Jackson, E., \& Risley, T. R. (1976). Contingent observation: An 
effective and acceptable procedure for reducing disruptive behavior of young children in a group setting. Journal of Applied Behavior Analysis, 9(1), 55-64.

Richardson, J.V., \& Baron, A. (2008). Avoidance of timeout from response-independent food: effects of delivery rate and quality. Journal of the Experimental Analysis of Behavior, 89, 169-181. doi: 10.1901/jeab.2008.89-169

Shriver, M. D., \& Allen, K. D. (1996). The time-out grid: A guide to effective discipline. School Psychology Quarterly, 11(1), 67. doi: 10.1037/h0088921

Solnick, J. V., Rincover, A., \& Peterson, C. R. (1977). Some determinants of the reinforcing and punishing effects of timeout. Journal of Applied Behavior Analysis, 10(3), 415-424. doi:10.1901/jaba.1977.10-415

Thomas, J. R. (1964). Avoidance of time-out from VI schedules of positive reinforcement. Journal of the Experimental Analysis of Behavior, 7, 168.

van Haaren, F., \& Anderson, K. G. (1998). Avoidance of time-out from response-independent food presentation: Effects of chlordiazepoxide and buspirone. Pharmacology Biochemistry and Behavior, 61, 207-214. doi: 10.1016/S0091-3057(98)00101-4

Van Houten, R., Axelrod, S., Bailey, J. S., Favell, J. E., Foxx, R. M., Iwata, B. A., \& Lovaas, O. I. (1988). The right to effective behavioral treatment. Journal of Applied Behavior Analysis, 21(4), 381. 